\title{
Inconsistency of quantum-classical dynamics, and what it implies
}

\author{
Daniel R. Terno \\ Perimeter Institute for Theoretical Physics, 35 King St. N., Waterloo, Ontario, Canada N2J 2W9
}

\begin{abstract}
A new proof of the impossibility of a universal quantum-classical dynamics is given. It has at least two consequences. The standard paradigm "quantum system is measured by a classical apparatus" is untenable, while a quantum matter can be consistently coupled only with a quantum gravity.
\end{abstract}

PACS numbers: 03.65.Sq, 03.65.Ta, 04.60-m

Quantum mechanics gives exceedingly accurate predictions for atomic and nuclear systems. Classical mechanics is just as successful for planetary motion. When it is necessary or convenient to describe part of the system classically and another part quantum mechanically both languages are combined. The phenomena that are treated by this mixed dynamics range form the one-loop quantum gravity [1] and quantum cosmology [2] to gas kinetics 3] and dynamics of chemical reactions [4]. Different mixed dynamics schemes are adopted for different situations. Often they produce a good agreement with experimental data or reasonable physical models, but these schemes are also prone to sudden breakdowns and require fine tuning of parameters or a careful selection of allowed initial states.

Quantum mechanics itself is dependent on such a coupling, because its basic notions are "preparations" and "tests" (measurements). They are performed by macroscopic devices and these are described in classical terms. Any intermediate systems used in that process could be treated quantum mechanically, but the final instrument has a purely classical description. The necessity of using a classical terminology was emphasized by Bohr [5], even though he never considered the measuring process as dynamical interaction between an apparatus and the system under observation. Yet, measuring apparatuses are made of the same kind of matter as everything else and they obey the same physical laws. It therefore seems natural to use quantum theory in order to investigate their behavior during a measurement. This was attempted by von Neumann [6]. Von Neumann represented the apparatus by a single degree of freedom, whose value was correlated to that of the dynamical variable being measured. Such an apparatus is not, in general, left in a definite pure state and does not admit a classical description. Therefore, von Neumann introduced a second apparatus, which observes the first one, and possibly a third apparatus, and so on, until there is a final measurement, which is not described by quantum dynamics and has a definite result. These different approaches of Bohr and von Neumann are reconciled by a dual description of the measuring apparatus. It obeys quantum mechanics while it interacts with the system under observation, and then it is "dequantized" and is described by a classical Liouville density which provides the probability distribution for the results of the measurement [7, 8].

Is it possible to consistently maintain a distinction be- tween a classical apparatus and a quantum system during all stages of the measurement process? Is it possible to consistently argue about the reaction of a quantized matter on a classical metric? Both answers depend on the existence of a general quantum-classical interaction scheme, as opposed to the different effective methods that were mentioned above. A universal mixed dynamics formalism should be similar to, e.g., the Hamiltonian formulation of classical and quantum mechanics, where the information about a system is provided by its Hamiltonian, which is then plugged into the standard scheme. Such universal formalism is impossible [9, 10]. In this paper we give a new derivation of this result from a minimal number of assumptions and explore its consequences. Koopmanian formalism 11, 12, 13] for classical mechanics illustrates both the desiderata of the measurement description and helps to derive the impossibility of the mixed dynamics in a concise form.

For simplicity we consider a single degree of freedom and denote the canonical variables as $x$ and $k$ (we reserve the symbol $p$ for the momentum of a quantum system, to be introduced later). Let us write the Liouville equation as

$$
i \partial f / \partial t=L f
$$

where $L$ is the Liouville operator, or Liouvillian,

$$
L=\left(\frac{\partial H}{\partial k}\right)\left(-i \frac{\partial}{\partial x}\right)-\left(\frac{\partial H}{\partial x}\right)\left(-i \frac{\partial}{\partial k}\right) .
$$

The Liouville density $f$ is never negative. It is convenient to introduce likewise a "classical wave function"

$$
\psi_{\mathrm{c}} \equiv \sqrt{f}
$$

which in this case satisfies the same equation of motion as $f$,

$$
i \partial \psi_{\mathrm{c}} / \partial t=L \psi_{\mathrm{c}}
$$

We shall now consider $\psi_{\mathrm{c}}$ as the fundamental entity (but only $f=\left|\psi_{\mathrm{c}}\right|^{2}$ has a direct physical meaning). It can be proved that, under reasonable assumptions about the Hamiltonian, the Liouvillian is an essentially self-adjoint operator and generates a unitary evolution [14]:

$$
\left\langle\psi_{\mathrm{c}} \mid \phi_{\mathrm{c}}\right\rangle:=\int \psi_{\mathrm{c}}(x, k, t)^{*} \phi_{\mathrm{c}}(x, k, t) d x d y=\text { const. }
$$


It is possible to further mimic quantum theory by introducing commuting operators $\hat{x}$ and $\hat{k}$, defined by

$$
\hat{x} \psi_{\mathrm{c}}=x \psi_{\mathrm{c}}(x, k, t) \quad \text { and } \quad \hat{k} \psi_{\mathrm{c}}=k \psi_{\mathrm{c}}(x, k, t) .
$$

Note that the momentum $\hat{k}$ is not the shift operator (the latter is $\hat{p}_{x}=-i \partial / \partial x$ ). Likewise the boost operator is $\hat{p}_{k}=-i \partial / \partial k$. These two operators are not observable. We shall henceforth omit the hats over the classical operators when there is no danger of confusion.

The analogy with quantum mechanics can be pushed further. What we have above is a "Schrödinger picture" (operators are constant, wave functions evolve in time as $\psi(t)=U(t) \psi(0)$, where $U(t)=e^{-i L t}$ if the Hamiltonian is time-independent). We can also define a "Heisenberg picture" where wave functions are fixed and operators evolve:

$$
X_{H}(t)=U^{\dagger} X U
$$

The Heisenberg equation of motion,

$$
i d X_{H} / d t=\left[X_{H}, L_{H}\right]=U^{\dagger}[X, L] U,
$$

together with the Liouvillian (2), readily provide Hamilton's equations

$$
\frac{d x}{d t}=\frac{\partial H}{\partial k}, \quad \frac{d k}{d t}=-\frac{\partial H}{\partial x} .
$$

This formalism allows to describe the states of classical and quantum systems in a single mathematical framework, namely in the joint Hilbert space $\mathcal{H}=\mathcal{H}_{\mathrm{q}} \otimes \mathcal{H}_{\mathrm{c}}$. Since we are dealing with the Hilbert spaces, the concepts of a partial trace and entanglement (including the one between classical and quantum states) are naturally defined.

A measurement apparatus produces a classical (i.e., robust, clonable) data. If the measurement interaction is sufficiently well understood, the resulting quantum state is given by a set of Kraus matrices 15. Consider any measurement that can result in a finite number of the discrete outcomes (this is what happens in any actual experiment). If a particular outcome $\mu$ is registered, then

$$
\rho_{\mid \mu}=\frac{1}{\operatorname{tr}\left(\rho E_{\mu}\right)} \sum_{i} A_{\mu i} \rho A_{\mu i}^{\dagger}
$$

where $\rho$ is the initial density matrix, a probability of the outcome $\mu$ is calculated as an expectation of a positive operator-valued measure (POVM) element $E_{\mu}$,

$$
p(\mu \mid \rho)=\operatorname{tr} \rho E_{\mu},
$$

and the POVM is constructed from the Kraus matrices $A_{\mu i}$,

$$
E_{\mu}=\sum_{i} A_{\mu i}^{\dagger} A_{\mu i}
$$

To literally accept the paradigm "quantum systemclassical apparatus", we need a description of dynamics that leads to the above probability formula and conditional evolution. It is well understood that the decoherence 16] plays an important role in the measurement process. Hence, in addition to the Hamiltonian interaction between system and apparatus an interaction with the environment should be taken into account. This interaction typically selects a preferred pointer basis and severely limits possible POVMs 16.

To mimic a quantum formalism, we describe classical measurements by projection operators $\Pi_{\mu}$. Since any Liouville density can be put into the form of a pure state by Eq. (3),

$$
\Pi_{\mu} \equiv\left|\psi_{\mathrm{c} \mu}\right\rangle\left\langle\psi_{\mathrm{c} \mu}\right|,
$$

where $\left\langle\psi_{\mathrm{c} \mu} \mid \psi_{\mathrm{c} \nu}\right\rangle=0, \mu \neq \nu$. This set may be taken as complete,

$$
\sum_{\mu} \Pi_{\mu}=\mathbb{1}
$$

For example, we can partition the phase space into domains $X_{\mu}$. Then the projectors may be taken as

$$
\Pi_{\mu}(x, k)=\chi_{\mu}(x, k),
$$

where $\chi_{\mu}$ is an indicator of the set $X_{\mu}$ (equals to 1 if $(x, k) \in X_{\mu}$ and is zero otherwise). Hence

$$
\operatorname{tr}\left(\Pi_{\mu}\left|\phi_{\mathrm{c}}\right\rangle\left\langle\phi_{\mathrm{c}}\right|\right)=\int_{X_{\mu}}|\phi(x, k)|^{2} d x d k
$$

It should be noted that projectors onto some orthogonal basis states of the classical Hilbert space do not necessary have a physical meaning. Similarly, while the state on, say, a two particle Hilbert space $\mathcal{H} \otimes \mathcal{H}^{\prime}$ may be written in the form $\psi_{12}=\sum_{i} \phi_{\mathrm{c}}^{i}(x, k) \phi_{\mathrm{c}}^{i^{\prime}}\left(x^{\prime}, k^{\prime}\right)$, the apparent entanglement is fictitious. It can't be detected by any measurement of the type of Eq. (15).

Now we can state how the measurement formalism should be derived. We follow the standard splitting of the Universe into a triple: the system - the apparatus - their environment [12, 16, 17]. The system is described by quantum mechanics, the apparatus by classical mechanics, and the environment may be anything. For classical systems we use the Koopmanian formalism. An initial state is

$$
\rho_{\mathrm{q}} \otimes \rho_{\mathrm{c}} \otimes \alpha,
$$

where $\alpha$ is an initial state of the environment. The overall evolution is unitary. It may entangle anything with anything else, but it is reversible. The irreversibility is introduced by tracing out the environment, which may also dictate the choice of the basis. An outcome $\mu$ of the measurement induces the state transformation

$\rho_{\mathrm{q} \mid \mu}=\frac{1}{p(\mu \mid \rho)}\left(\mathbb{1}_{\mathrm{q}} \otimes \Pi_{\mu}\right) \operatorname{tr}_{\mathrm{env}^{\prime} \mathrm{t}}\left[U\left(\rho_{\mathrm{q}} \otimes \rho_{\mathrm{c}} \otimes \alpha\right) U^{\dagger}\right]\left(\mathbb{1}_{\mathrm{q}} \otimes \Pi_{\mu}\right)$, 
and by comparison with Eqs. (10)-(12) the POVM and Kraus matrices can be identified.

If we ignore the environment, the (pre-)measurement 12 process may be described as follows. Initially the combined system is in the state

$$
\left|\Psi^{0}\right\rangle=\left|\psi_{\mathrm{q}}^{0}\right\rangle \otimes\left|\psi_{\mathrm{c}}^{0}\right\rangle
$$

After some unitary interaction, the combined system is in the state

$$
\left|\Psi^{1}\right\rangle=\sum_{\mu} \alpha_{\mu}\left|\psi_{\mathrm{q} \mu}^{1}\right\rangle \otimes\left|\psi_{\mathrm{c} \mu}^{1}\right\rangle
$$

where $\sum_{\mu}\left|\alpha_{\mu}\right|^{2}=1$ and classical Liouville densities $f_{\mu} \equiv\left|\psi_{\mathrm{c} \mu}^{1}(x, k)\right|^{2}$ are distinguishable, $\left\langle\psi_{\mathrm{c} \mu}^{1} \mid \psi_{\mathrm{c} \nu}^{1}\right\rangle=0$, $\mu \neq \nu$. (Several projectors may be lumped together to form a single $\Pi$ ). Then if the outcome $\mu$ is found then the wavefunction of the system "collapses" to $\psi_{\mathrm{c} \mu}^{1}$. A realization of this scheme depends on the possibility to produce the unitary $U$ that is responsible for the interaction of classical and quantum subsystems.

Koopmanian formalism automatically takes care of the two basic requirements for mixed dynamics. First, in the absence of interaction the systems split into classical and quantum parts that evolve according to the rules of the corresponding mechanics. Second, expectation values of positive quantum operators and classical probability densities are positive. This allows a simple derivation of the no-go result $([9,10]$ and references therein) with the minimal number of assumptions.

We assume the following:

- Quantum sector is described by usual quantum mechanics.

- Classical sector is described by Koopmanian dynamics, with $x$ and $k$ promoted to commuting multiplication operators.

- A dynamics of the combined system is described by a unitary evolution on the joint Hilbert space $\mathcal{H}=\mathcal{H}_{\mathrm{q}} \otimes \mathcal{H}_{\mathrm{c}}$ that is given by some unitary operator $U$. The generator of the interaction part is $K_{i}$. This is our definition of the mixed dynamics.

It follows from these definitions that classical and quantum operators commute, in particular

$$
[q, x]=[p, k]=\left[p, p_{x}\right]=\ldots=0 .
$$

This is just a standard result for operators that act on different Hilbert spaces. The existence of the unitary dynamics on the combined Hilbert spaces ensures that these relations hold all the time.

First, we assume that the interaction term $K_{i}$ contains only observables, i. e., $x, k, q$ and $p$. Then the commutativity of the classical observables with addition of Eq. (21) isolates the classical degrees of freedom from the quantum ones,

$$
\left[x, K_{i}\right]=\left[k, K_{i}\right]=0 .
$$

This result does not forbid a more complicated interaction term that involves also operators $p_{x}$ and $p_{k}$. Indeed, it is possible to construct such terms [10]. However, to ensure that a quantum sector influences classical observables, an interaction $K_{i}\left(p_{x}, p_{k}, x, k, q, p\right)$ should have terms of the form $q p_{x}, p p_{k}$ and/or similar ones. Thus the equations for $q$ and/or $p$ will acquire terms with classical unobservable operators, thus becoming different from both classical and quantum equations of motion. If formal correspondence with either purely classical or purely quantum equations of motion is a benchmark that is used to judge the validity of mixed dynamics, then it fails the test.

Correspondence with the classical or quantum equations is more than just a formal requirement. If it is violated, there will be an inconsistency with a classical limit, as we now explain. When the analysis is not restricted to the commuting variables, classical behavior means that expectation values of operators $\hat{q}(t)$ and $\hat{p}(t)$ tend to the expectation values of classical dynamical variables $q(t)$ and $p(t)$ that are calculated from an appropriate Liouville density.

More precisely [18], POVMs that describe a joint measurement of position and momentum result in probability distributions

$$
f(p, q, t) d p d q=\operatorname{tr}[d E(p, q) \rho(t)],
$$

that go to the classical Liouville probability density $f_{\mathrm{c}}(p, q, t)$ that evolves as in Eq. (11). Here a POVM for the joint position-momentum measurement satisfies $\int d E(p, q)=1$, and $\rho$ is system's density matrix. Moreover, we expect that

$$
\operatorname{tr}(\hat{O}(t) \rho)=\langle O(t)\rangle=\int O f(p, q, t) d q d p,
$$

where $O=q, p$, while the illusion of "sharpness" and "infinite precision" of classical theory are achieved by

$$
\Delta q /\langle q\rangle \ll 1, \quad \Delta p /\langle p\rangle \ll 1 \quad \Delta q \Delta p \gg \hbar
$$

holding for all times. All these statements should be qualified: the limits are expected to hold for the "reasonable" systems and "appropriate" POVMs. Analysis of quantum-to-classical transitions is a subject of current research. However, for simple systems like two harmonic oscillators, with a bilinear coupling $c q x$, where $c$ is a constant, the correspondence is easily established. If we treat both of them classically, with a Hamiltonian

$$
H=\frac{1}{2}\left(q^{2}+p^{2}+x^{2}+k^{2}\right)+c q x
$$

we obtain equations of motion

$$
\begin{array}{ll}
\dot{q}=p, & \dot{p}=-q-c x, \\
\dot{x}=k, & \dot{k}=-x-c q .
\end{array}
$$


Exactly the same equations of motion appear in the Heisenberg picture for quantum mechanics.

Now if for some of the variables the Koopmanian equation of motion $\dot{O}=\ldots$ differs from the above, the limit of Eqs. (23) and (24) will break down [19], since

$$
O(t)=O(0)+t \dot{O}(0)+\ldots
$$

and the expectation value $\langle O(t)\rangle, t>0$ will be different from its classical counterpart even if they agree at $t=0$. Thus we see that a formal identity of the equations is necessary for the classical limit to hold. However, $K_{i}$ that is built only from observables cannot produce the interaction terms, while a more general term cannot mimic the classical (or quantum) equations for observables. It will add additional terms into the equation for unobservable operators $p_{x}$ and $p_{k}$, which in turn will change the equations for observables [10]. For some systems, like the one given by Eq. (26), an introduction of the the mixed dynamics leads to even more bizarre consequences. If the interaction term of the Hamiltonian, $c q x$ is replaced by the most natural Koopmanian term, $c q p_{k}$, it results in the infinite flow of energy from the classical to the quantum oscillator [10].

This discussion can be summed as follows. To have a non-trivial quantum-classical dynamics it is necessary to have classical non-observable operators in the interaction term, but their presence leads to the violation of correspondence principle and may result in energy nonconservation.

As a result, it is impossible to maintain that apparatus is described by classical mechanics through the interaction. The "dual" approaches, where the apparatus is treated either quantum-mechanically or classically [7, 8] are necessary.

Since the classical gravity (pure or with matter) can be formulated in the Hamiltonian form [20], the above result applies to it as well. Impossibility of the universal mixed dynamics means that to get a fully consistent description of the gravitation phenomena, there must be a quantized theory of gravity. This analysis shows that while in each particular case there is a specific reason for the inconsistencies or a necessity of fine tuning, no approach that mixes a quantized matter (or fluctuating cosmological constant) and a classical gravity is fully consistent. Moreover, this adds another question [21] to the validity of the black hole radiation and their eventual evaporation. The radiation is derived as an effect of a quantum field theory on a fixed curved background. The black hole evaporation is concluded from the analysis of the backreaction of the quantum fields on the classical metric [22], which is inconsistent. However, a conclusive resolution of this problem will be possible only after a full theory of quantum gravity emerges.

\section{Acknowledgments}

Useful discussions with Daniel Gottesman, Netanel Lindner, John Moffat, Asher Peres, Lee Smolin, Rob Spekkens, and Rafael Sorkin are gratefully acknowledged.
[1] C. Möller, in Les Théories Relativistes de la Gravitation, (Centre National de la Recherche Scientifique, Paris, 1962) p. 15; L. Rosenfeld, Nucl. Phys. 40353 (1963); A. Peres, Nucl. Phys. 48, 622 (1963); B. S. DeWitt, in General Relativity: an Einstein Centenary Survey, edited by S. W. Hawking and W. Israel (Cambridge University Press, Cambridge, 1979) p. 680; W. Boucher and J. Traschen, Phys. Rev. D 37, 3522 (1988).

[2] J. N. Islam, An Introduction to Mathematical Cosmology (Cambridge University Press, Cambridge, 2002); M. Ahmed, S. Dodelson, P. B. Greene, R. Sorkin, e-print astro-ph/0209274

[3] P. Pechukas, Phys. Rev. 181, 166 (1969); I. V. Aleksandrov, Z. Naturforsch. 36A, 902 (1981).

[4] B. J. Schwartz, E. R. Bittner, O. V. Prezhdo, and P. J. Rossky, J. Chem. Phys. 104, 5942 (1996); K. Ando and M. J. Santer, J. Chem. Phys. 118, 10399 (2003).

[5] N. Bohr, Nature 121, 78, 580 (1928); N. Bohr, in Albert Einstein: Philosopher-Scientist, edited by P. A. Schilpp, (1949) p. 199.

[6] J. von Neumann, Mathematical Foundations of Quantum Mechanics, (Princeton University Press, Princeton, 1955).

[7] O. Hay and A. Peres, Phys. Rev. A 58, 116 (1998).

[8] L. Diósi, N. Gisin, W. T. Struntz, Phys. Rev. A 61, 022108 (2000).
[9] L. L. Salcedo, Phys. Rev. A 543657 (1996); J. Caro and L. L. Salcedo, Phys. Rev. A 60, 842 (1999).

[10] A. Peres and D. R. Terno, Phys. Rev. A 63, 022101 (2001).

[11] B. O. Koopman, Proc. Nat. Acad. Sci. 17, 315 (1931).

[12] A. Peres, Quantum Theory: Concepts and Methods (Kluwer, Dordrecht, 1995), p. 312.

[13] D. Mauro, Topics in Koopman-von Neumann Theory, Ph.D. thesis (Università di Trieste, 2002); e-print quant-ph/0301172

[14] M. Reed and B. Simon, Methods of Modern Mathematical Physics, vol. 1, Functional Analysis (Academic Press, New York, 1972); vol. 2, Fourier Analysis, SelfAdjointness (Academic Press, New York, 1975).

[15] E. B. Davies, Quantum Dynamics of Open Systems (Academic Press, New York, 1976); A. S. Holevo, Probabilistic and Statistical Aspects of Quantum Theory (NorthHolland, Amsterdam, 1982); K. Kraus, States, Effects, and Operations: Fundamental Notions of Quantum Theory (Springer, Berlin, 1983).

[16] W. H. Zurek, Rev. Mod. Phys. 75, 715 (2003).

[17] A. Peres, Phys. Rev. A 61, 022116 (2001).

[18] P. Busch, M. Grabowski, and P. J. Lahti, Operational Quantum Physics (Springer, Berlin, 1995), ch VI.3.

[19] L. E. Ballentine and S. M. McRae, Phys. Rev. A 58, 1799 (1998). 
[20] R. M. Wald, General Relativity (University of Chicago, Chicago, 1984); A. Ashtekar, Lectures on nonperturbative canonical gravity (World Scientific, 1991); T. Thiemann, Introduction to Modern Canonical Quantum General relativity, e-print gr-qc/0110034 (2001).
[21] A. D. Helfer, Rep. Prog. Phys. 66, 943 (2003).

[22] Brout, R., S. Massar, R. Parentani, Ph. Spindel, Phys. Rep. 260, 329 (1995); V. P. Frolov and I. D. Novikov, Black Hole Physics (Kluwer, Dordrecht, 1998). 